\title{
Wra 751, a luminous blue variable developing an S Doradus cycle ${ }^{\star}$
}

\author{
C. Sterken ${ }^{1, \star \star}$, A. M. van Genderen ${ }^{2}$, A. Plummer ${ }^{3}$, and A. F. Jones ${ }^{4}$ \\ 1 Vrije Universiteit Brussel, Pleinlaan 2, 1050 Brussels, Belgium \\ e-mail: csterken@vub.ac.be \\ 2 Leiden Observatory, Postbus 9513, 2300RA Leiden, The Netherlands \\ 3 Linden Observatory, 105 Glossop Rd. Linden 2778, Australia \\ 4 Ranui Road, Nelson, New Zealand \\ Received 11 March 2008 / Accepted 25 March 2008
}

ABSTRACT

\begin{abstract}
Aims. The object Wra 751 is a luminous blue variable that lately exhibits strong changes in light and color. We summarise the available photometry of Wra 751, present new photometric observations, and discuss these data with special attention on the systematic differences between the various data sources. In addition, we establish an empirical relationship between $b-y$ and $B-V$ for this class of stars.

Methods. Wra 751 is a strong-active member of the S Dor class, and it exhibits very-long term S Doradus phases with an amplitude of about two magnitudes in $V$ and a cycle length of several decades. The associated $B-V$ colour-index amplitude is about 0.4 mag. At this moment this LBV, which is the reddest member of the class, is going through the bright (and red) stage of a long-term $\mathrm{S}$ Dor cycle.

Results. The S Dor behaviour of this system shows some resemblence to the temporal characteristics of the Galactic LBV AG Car, because the time scales and amplitudes of light and colour variability are very similar.
\end{abstract}

Key words. stars: variables: general - stars: individual: general - stars: early-type - stars: supergiants - techniques: photometric

\section{Introduction}

The source Wra 751 (V432 Car, $V=10.5-12.5$ ) is a member of the class of $\mathrm{S}$ Doradus variables - a group with about 50 known members, commonly called luminous blue variables (LBVs). These are evolved massive stars that display four major types of intrinsic photometric variability: microvariations, S Doradus phases, stochastic variability, and eruptions. For a detailed discussion of these types of variabilities and for a very complete review of the state of affairs at the end of the second millennium, we refer to van Genderen (2001). A revised census of galactic LBVs was published by Clark et al. (2005), but see also Humphreys \& Stanek (2005) for the proceedings of the latest conference on $\mathrm{LBV}$ s.

Wra 751 - like LBVs AG Car and HR Car located in the Galactic Carina arm - is one of the two known Galactic LBVs associated with its birth cluster (Pasquali et al. 2006). Over the past decade, the star has brightened by almost two magnitudes in the visible part of the spectrum. Its spectral type was O $9.5 \mathrm{I}$ in 1989 (Hu et al. 1990), and the star now has a much redder visual appearance.

\section{The data}

\subsection{Archival UBV photometric data}

The earliest visual magnitude of Wra $751(V=10.7 \pm 0.6-$ as cited by Henize 1976) was obtained in 1949-1950 by Wray

\footnotetext{
* Individual photometric measurements (Tables 2-4) are only available in electronic form at the CDS via anonymous ftp to cdsarc.u-strasbg.fr (130.79.128.5) or via http://cdsweb.u-strasbg.fr/cgi-bin/qcat?]/A+A/484/463

$\star \star$ Research Director, Belgian Fund for Scientific Research.
}

during his PhD work (Wray 1966). Henize (1976) mentioned that this object is a P Cygni star with $V=9.8 \pm 0.4$. Both magnitudes have a very uncertain time stamp.

Hu et al. (1990) obtained $V=12.43, B-V=1.38$, and $V=12.21, B-V=1.71$ on two consecutive nights in March 1989 (see Fig. 1), and also mention an unpublished $V=11.79$ obtained by $\mathrm{O}$. Stahl in 1984 . The $0^{\mathrm{m}} .2$ and $0^{\mathrm{m}} .3$ difference in $V$ and $B-V$ (connected points in Fig. 1) from one night to the next is unlikely to be real.

\subsection{Walraven photometry}

Van Genderen et al. (1992) discussed Walraven VBLUW photometry of the Wra 751 field, and also presented light- and colour curves of the object. We have taken the Walraven differential photometry (intensity scale, relative to the comparison star HD 96946) from Table 3 of van Genderen et al. (1992), and transformed the magnitudes and indices to their Johnson $V, B-V$ equivalents using a transformation formula from Pel (1987). For the comparison star HD 96946 we used $V=8.473, B-V=$ 0.224 from Klare \& Neckel (1977). The results are plotted in Fig. 1. The derived Johnson $V$ time-series reveals a $V=12.25$ minimum around JD 2444800 (1990), about the same light level as reported by $\mathrm{Hu}$ et al. (1990). Table 2 lists the Walraven photometry.

\subsection{ASAS data}

The $V$ magnitudes were extracted from the All Sky Automated Survey (ASAS) Catalog Sky Atlas (data coverage 2001-2006), and the following criteria were applied. Only magnitudes of grade A and aperture 0 were used, and only those fields were retained where magnitudes of both Wra 751 and HD 96946 


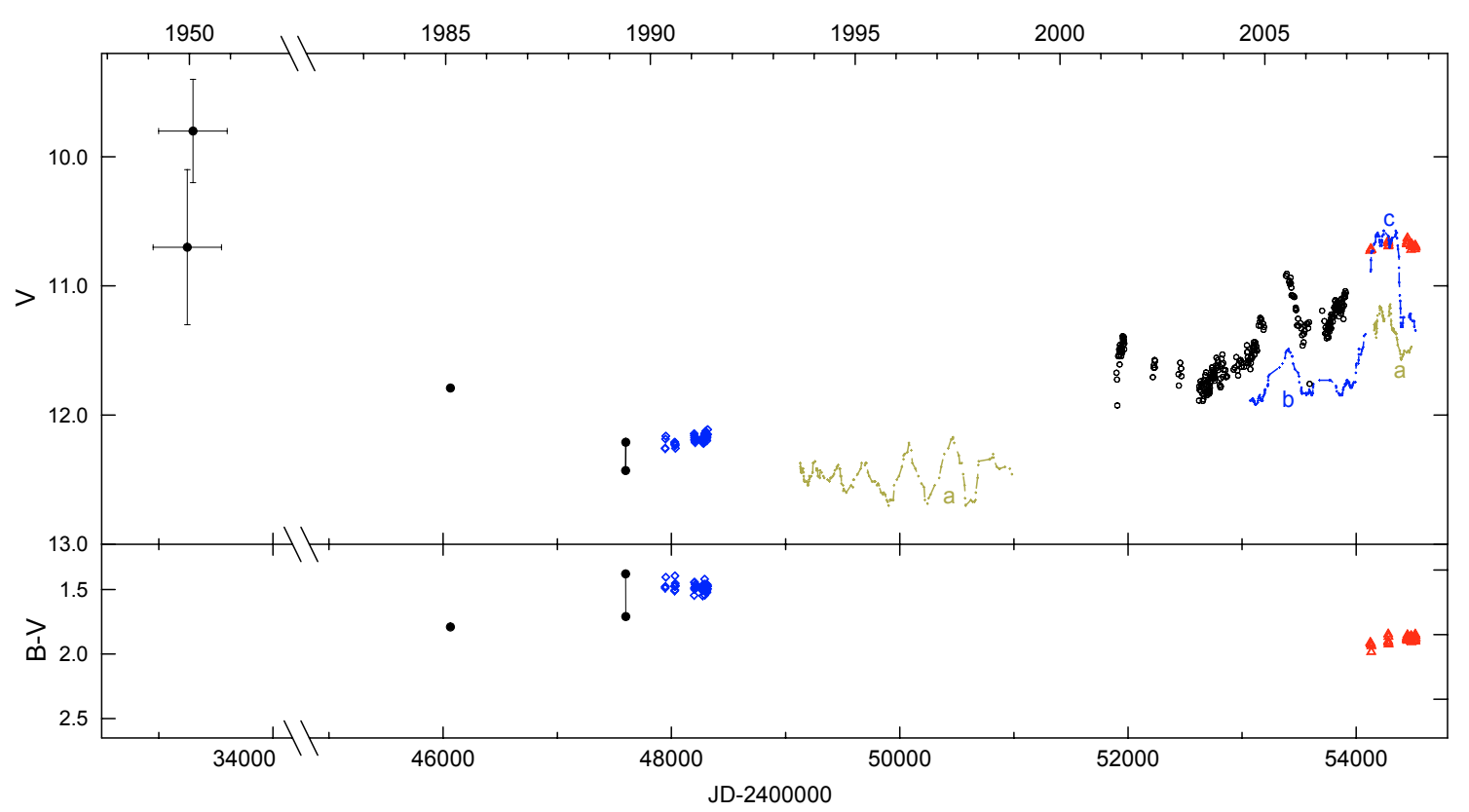

Fig. 1. $V, B-V$ variability of Wra 751. $\bullet$ : archival data described in Sect. 2.1 (error bars are estimated mean errors). $\diamond$ : van Genderen et al. (1992); $\circ$ : ASAS magnitudes; $\triangle$ : this paper. Visual estimates are represented as running averages: $a$ (AFJ 32-cm), $b$ (AP 20-cm), $c$ (AP 30-cm).

were extracted from the same CCD frame. In the next step, all HD $96946 V$ magnitudes deviating by more than 0.04 from the mean were eliminated. This resulted in $V=8.47 \pm 0.01$ for the 227 mag of ASAS Batch 1, and $V=8.48 \pm 0.02$ for the 55 mag of Batch $2^{1}$. The final $V$ magnitudes of Wra 751 were derived by subtracting these Batch 1 and Batch 2 zeropoints, and by adding the $V=8.473$ from Klare \& Neckel (1977). The Wra 751 ASAS data appear as open circles in Fig. 1. The ASAS- $V$ and the Strömgren $y$ derived above are in very good accord with the results of Klare \& Neckel (1977). Table 3 lists the ASAS $V$ photometry.

\subsection{Strömgren photometry}

The system was observed with the Strömgren Automatic Telescope (SAT) at ESO, La Silla on several occasions in January, July, and December 2007, and in January and February 2008. A diaphragm of 17 arcsec was used. Extinction corrections were derived from standard-star observations. Transformation relations to the standard uvby system were obtained during 19 nights yielding the following photometric magnitudes and indices for HD 96946: $V=8.470 \pm 0.001, b-y=$ $0.233 \pm 0.001, m_{1}=-0.034 \pm 0.001, c_{1}=-0.141 \pm 0.003$. Our data show that HD 96946 has rather constant Strömgren colour indices $^{2}$ (see also Shobbrook \& Lynga 1994; Knude 1992).

Differential magnitudes and colour indices of Wra 751 relatively to HD 96946 were calculated, and these photometric data were then transformed into the standard uvby following Olsen (1994), using averaged transformation coefficients obtained from all nights with well-determined transformation equations. This leads to an average $V=10.689 \pm 0.004$ for Wra 751, with $b-y=1.530 \pm 0.005, m_{1}=-0.473 \pm 0.010$, $c_{1}=0.429 \pm 0.030$. As Wra 751 is extremely red $^{3}$ and very faint

\footnotetext{
1 ASAS data are delivered in subsets that depend on slightly different calibrations.

2 By definition, $m_{1}=(v-b)-(b-y)$ and $c_{1}=(u-v)-(v-b)$.

${ }^{3}$ Note that the red colour of Wra 751 is mostly due to interstellar and circumstellar extinction.
}

in the $u$ band, its $u v$ colour indices fall outside the range of standard star colour indices, hence the indices $m_{1}$ and $c_{1}$ have substantial error ranges. Figure 1 includes the $y$ magnitudes transformed to the Johnson $V$ scale. Table 4 lists the Strömgren $y$, $b-y$ and transformed $B-V$ photometry.

\subsection{Visual photometric estimates}

Visual estimates were collected by AFJ (Newtonian 32-cm telescope, latitude $-41^{\circ}$ ) and consist of two series, collected in 1994-1998 and from 2007 on ( $a$ in Figs. 1 and 2). The visual magnitudes result from comparing the variable with one brighter and one fainter comparison star, and the estimate of the brightness of the variable star is then made by the fractional method. No estimates of our comparison star HD 96946 were obtained, as the magnitude difference of $\sim 2 \mathrm{~m} .5$ with Wra 751 makes this comparison star inadequate for visual work.

The 1994-1998 sequence displays two prominent features: there is an oscillation with a characteristic time of about one year, and the average magnitude is the lowest value ever seen. Both features must not be taken at face value, however, because the cyclic pattern is also present in visual estimates of other bright stars and is the consequence of unaccounted extinction corrections (Jones \& Sterken 1997) during the annual cycle of the data sampling invoking airmasses 3.0 and higher. The lower average $m_{V}$ may very well be due to a systematic difference $m_{V}-V$ of the order of 0.2 , as also present in the data published by Jones \& Sterken (1997).

Another set of magnitude estimates was collected by AP (20 and $30-\mathrm{cm}$ telescopes, latitude $-29^{\circ}$ ). These data have been obtained since 2004 (see b,c in Figs. 1 and 2) in a similar way. One estimate of HD 96946 (using an appropriate pair of comparison stars) was made for checking the observational procedure. Substantial systematic differences also occur between the visual estimates obtained with the $20-\mathrm{cm}$ telescope ( $b$ in Fig. 2 ) and the 30-cm instrument ( $c$ in Fig. 2). 


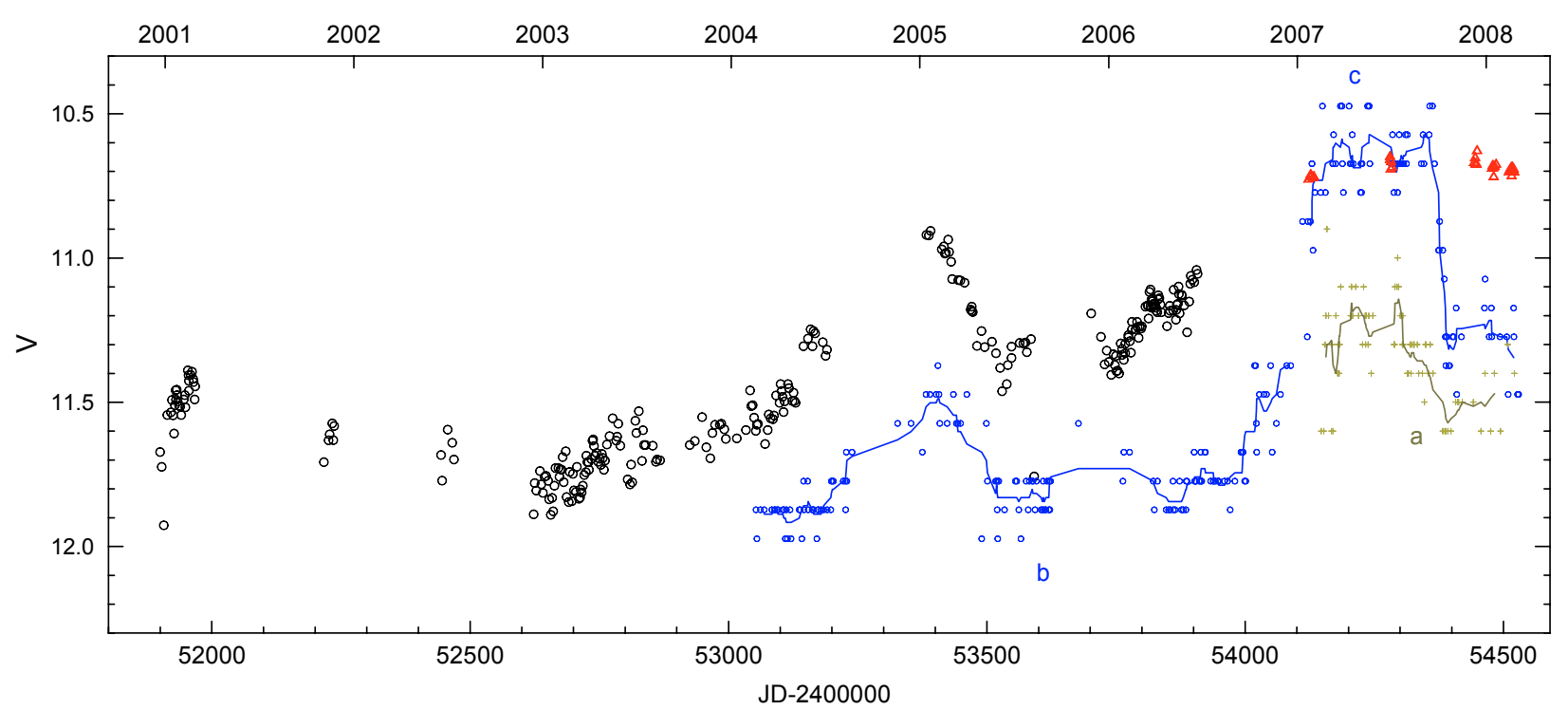

Fig. 2. The evolution of $V$ magnitude during the recent brightening (symbols as in Fig. 1).

\section{Discussion}

\subsection{The magnitudes}

The first point of discussion is the internal consistency of the data shown in Fig. 1: all photometric data-blocks appear to be quite coherent (the error bars of the photoelectric and CCD data are close to the size of the symbols), and the visual estimates mimic the trends quite closely, although they demonstrate significant systematic zero-point deviations. In particular, the estimates obtained by the two visual obsevers differ by 0.2 to 0.5 in 2007-2008. These large and variable differences between both data sets result from the combination of the systematic offset between both observers, and the colour effects caused by observing at high airmasses (observer AP worked at systematically higher airmasses than observer AFJ from JD 2454360 on, including occurrences of airmasses exceeding 4.0-5.0).

Part of the discrepancies between the magnitude sets (i.e. $y$, $V$, Walraven magnitudes transformed to $V$, and $m_{V}$ ) shown in Figs. 1 and 2 can be ascribed to the very different effective wavelengths of the "instrumental" bandpasses: the human eye's response in scotopic vision peaks at $\sim 510 \mathrm{~nm}$, whereas the $V$ band peaks near $\sim 530 \mathrm{~nm}$. In addition, the eye's response curve includes $\mathrm{H} \beta$ and is much wider than $V$, whereas the $V$ - unlike $y$ - includes $\mathrm{H} \alpha$ in its red tail ${ }^{4}$ (see Fig. 3). As both Balmer lines undergo prominent changes during the LBV variability cycles, their effect on the magnitude estimates are hard to quantify. In addition, there are quite a number of FeII and [FeII] emission lines all over the visual spectrum, (see Fig. 1 in Hu et al. 1990). These authors estimate the effect of these emission lines to 0.03 in the $V$ passband, although the differences between the $y$ and $V$ photometric scales for LBVs may easily reach 0 . 2 (for example in the case of $\eta$ Car, see Sterken et al. 1999). Figure 2 also reveals a remarkable discrepancy between the relatively stationary $V$ magnitude of Wra 751 (based on the differential $y$ with respect to HD 96946) and the visual estimates, viz. the 0.5 drop observed by AP in November 2007, and the 0.2 decrease as seen by AFJ.

That each set of visual estimates was made with instruments with different apertures, eyepieces, and detectors makes it very

\footnotetext{
${ }^{4}$ The red tail of the Johnson $V$ band is defined by the response of the photomultiplier and not by the filter.
}

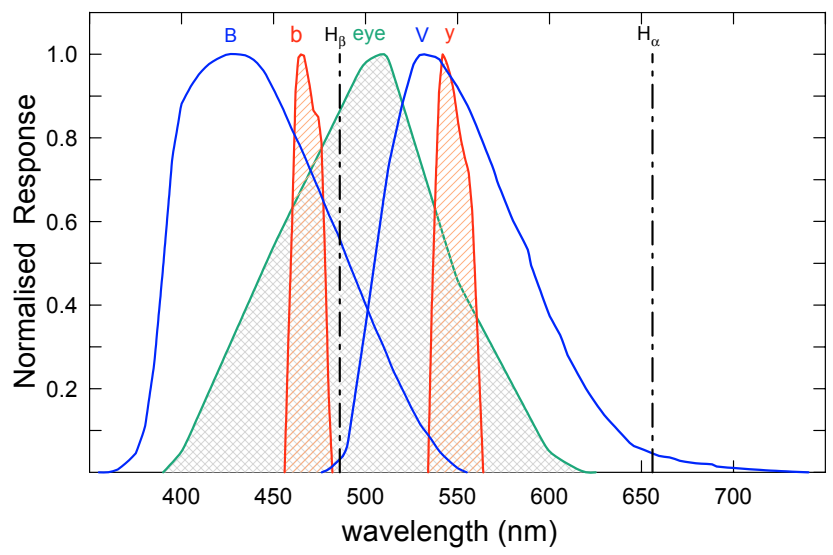

Fig. 3. Normalised photometric response of the $B, V, b$, and $y$ photometric bands. The response of the human eye in scotopic vision is also shown, as well as the location of the strong and variable Balmer emission lines.

difficult to combine these data - which span a time interval of 15 years - in one set of homogeneous quality. This aspect is quite often forgotten when combining undocumented visual estimates with wide-band and intermediate-band photometry.

\subsection{Estimating the $B-V$ colour index}

A generic $b-y$ to $B-V$ transformation (for example Eq. (51) of Caldwell et al. 1993) cannot be applied here because the very red $b-y$ colour of Wra 751 falls way beyond the validity interval of that relationship. We therefore compiled all available $b-y$ and $B-V$ indices of the strong-active, weak-active, and ex- (and dormant) LBVs listed by van Genderen (2001), and constructed the relationship (see Fig. 4)

$$
\begin{aligned}
& B-V=1.291 \quad(b-y)-0.075 \\
& \pm 0.023 \quad \pm 0.014
\end{aligned}
$$

valid for $0.1 \leq b-y \leq 1.4$. Table 1 lists the average colour indices and the sources of these data. All colour indices referred to in SIMBAD were considered, irrespective of the S Dor status of the object. As such, Eq. (1) should not be considered as a 


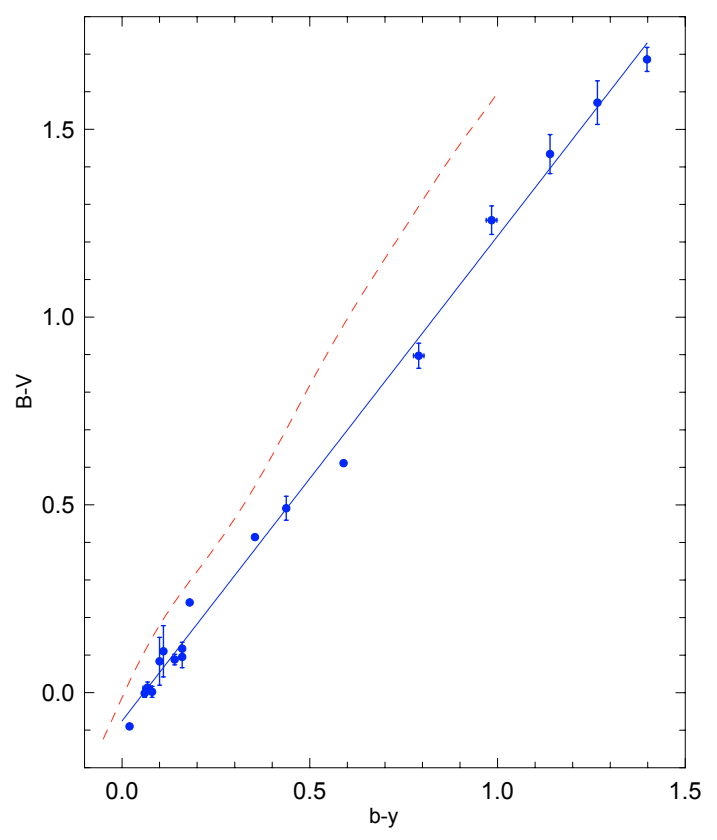

Fig. 4. $b-y, B-V$ relation for LBVs. The dashed line represents Eq. (51) of Caldwell et al. (1993), the full line is Eq. (1). Error bars represent two times the error of the mean.

photometric transformation in the true sense, but as a statistical relationship between the observables $b-y$ and $B-V$. Figure 4 displays a smaller variance in $b-y$ than in $B-V$ : the narrower range of variability is attributable to the smaller passband width of the uvby system.

\subsection{The light and colour curves of the LBV}

Figure 1 shows a typical pattern for LBVs: after a slow descent to a minimum $V$ brightness (associated with bluest colour), the system gradually becomes brighter and simultaneously redder. Such a cyclic pattern is called an "S Dor cycle", and we refer to van Genderen et al. (1997) for a discussion of the S Dor phenomenon in AG Car, S Dor, and $\eta$ Car.

The magnitude of the star and its $b-y$ and $m_{1}$ colour indices are quite stable over the last $\sim 500$ days (except for a brightening by 0.04 ), but the $c_{1}$ index decreased by $\sim-0.2$, pointing to an additional activity in the $u$ band. Such an effect was reported by Sterken (1977) for the first time in the case of HD 160529, which exhibited a slightly smaller ( $\sim$ m 20) blueing of its $u-v$ in step with a brightening of $\sim 0$ m 05 in $V$ in less than two months in 1972.

\subsection{Variability time scales}

In the case of Wra 751, it appears that the rising branch of the $\mathrm{S}$ Dor cycle, so far, takes about 5 years. The minimum phase is of similar duration, but the available data do not allow us to say whether the descending branch is about one decade, or perhaps 4-5 times as long. The large gap without data between 1950 and 1985 could very well be hiding a full S Dor cycle. Thus, the S Dor cycle-length of Wra 751 is comparable in amplitude and time scale to S Dor (35 years, 1-2 mag) and to AG Car (25 years, see van Genderen et al. 1997).

The 2003-2006 rising branch has a slope of $\sim 0 \mathrm{~m} 15 \mathrm{mag} \mathrm{yr}^{-1}$. Detrending the $V$ light curve with this slope, one can easily distinguish one relatively long wave with a cycle time of $\sim 640 \mathrm{~d}$ and
Table 1. Average $B-V$ and $b-y$ for LBVs.

\begin{tabular}{lllll}
\hline \hline ID & $B-V$ & $\operatorname{Ref}_{B-V}^{*}$ & $b-y$ & $\operatorname{Ref}_{b-y}^{*}$ \\
\hline AG Car & 0.611 & $24,26,33$ & 0.59 & 8 \\
HR Car & 0.897 & $12,26,33$ & 0.79 & 8,11 \\
HD 160529 & 1.258 & $1,26,29,31$ & 0.984 & 7,11 \\
R 4 & 0.095 & $22,25,26,32$ & 0.16 & 4 \\
R 40 & 0.088 & $4,14,15,25,26,28$ & 0.14 & 4 \\
R 71 & 0.110 & $4,15,18,21,23$ & 0.11 & 5 \\
S Dor & 0.083 & $15,18,26$ & 0.1 & 5 \\
R 110 & 0.240 & 15,23 & 0.18 & 5 \\
R 116 & 0.013 & $4,15,18,19$ & 0.068 & 5,9 \\
P Cyg & 0.414 & $1,7-8,16$ & 0.354 & 3 \\
HD 168607 & 1.571 & 1,34 & 1.266 & 7,12 \\
R 81 & 0.002 & $4,15,18-20$ & 0.08 & 5 \\
R 123 & 0.117 & $4,15,18,19$ & 0.16 & 5 \\
$\zeta^{1}$ Sco & 0.491 & $2,3,5,6,10,11,13$ & 0.437 & $1,2,6,7$ \\
& & $17,26,27,31,34$ & & \\
HD 168625 & 1.434 & 1,34 & 1.140 & 7,12 \\
HD 316285 & 1.686 & 29,33 & 1.398 & 10 \\
Sk 61 & -0.002 & $22,26,28,30$ & 0.06 & 4 \\
Sk 119 & -0.090 & $22,25,27$ & 0.02 & 4 \\
\hline
\end{tabular}

* References (in chronological order) for the data as listed in SIMBAD. References $B-V$ : 1: Hiltner (1956); 2: Hogg (1958); 3: Westerlund (1959); 4: Feast et al. (1960); 6: Walraven \& Walraven (1964); 7: Johnson (1964); 8: Johnson (1965); 9: Johnson et al. (1966); 10: Feinstein (1968); 11: Feinstein \& Ferrer (1968); 12: Feinstein (1969); 13: Schild et al. (1969); 14: Dachs (1970); 15: Mendoza (1970); 16: Crawford et al. (1971c); 18: Appenzeller (1972); 19: Ardeberg et al. (1973); 20: Lucke (1973); 21: Brunet et al. (1973); 22: Azzopardi et al. (1975); 23: Isserstedt (1975); 24: Deutschman et al. (1976); 25: Ardeberg \& Maurice (1977); 26: Nicolet (1978); 27: Feinstein \& Marraco (1979); 28: Ardeberg (1980); 29: Dachs (1982); 30: Vigneau \& Azzopardi (1982); 31: Fernie (1983); 32: Nandy et al. (1984); 33: Kilkenny et al. (1985); 34: Kozok (1985). References $b-y$ : 1: Crawford et al. (1970); 2: Crawford et al. (1971a); 3: Crawford et al. (1971b); 4: Osmer (1973a); 5: Osmer (1973b); 6: Gronbech \& Olsen (1976); 7: Sterken (1977); 8: Kilkenny et al. (1985); 9: Shobbrook (1986); 10: Manfroid et al. (1987); 11: Gray \& Olsen (1991); 12: Kilkenny \& Whittet (1993).

a range of $\sim 0$. 8 , and several smaller peaks of $\sim 0$. 1 with an average short cycle time of $\sim 55 \mathrm{~d}$. As there are no colour-index data available for that time interval, one cannot conclude whether the long cycles are microvariations, or else "normal" S Dor variations comparable to the AG Car $\sim 371 \mathrm{~d}$ variations (see Fig. 1 of van Genderen et al. 1997).

\subsection{The observational HR diagram}

Figure 5 illustrates the extreme positions of Wra 751 in the $\log L / L_{\odot}-\log T_{\text {eff }}$ diagram. The leftmost position corresponds to the lower state in 1989 with $T_{\text {eff }}$ and $\log L / L_{\odot}$ taken from Pasquali et al. (2006). The rightmost location is based on parameters derived using the distance of $6 \mathrm{kpc}$ from Pasquali et al. (2006) combined with $E_{B-V}=1.8$ from Hu et al. (1990) (bolometric corrections and $T_{\text {eff }} / B-V$ scale for supergiants from Flower 1996). The $S$ Dor-triangle in which the strongactive $\mathrm{S}$ Dor variables move to-and-fro during the $\mathrm{S}$ Dor-phases, the S Dor-minimum strip and the Yellow Void (de Jager \& Nieuwenhuijzen 1997; de Jager 1998) are also shown. The extreme positions of AG Car $\left(T_{\text {eff }}\right.$ and $\log L / L_{\odot}$ from van Genderen 2001) are also indicated, as well as evolutionary tracks for 25 , 


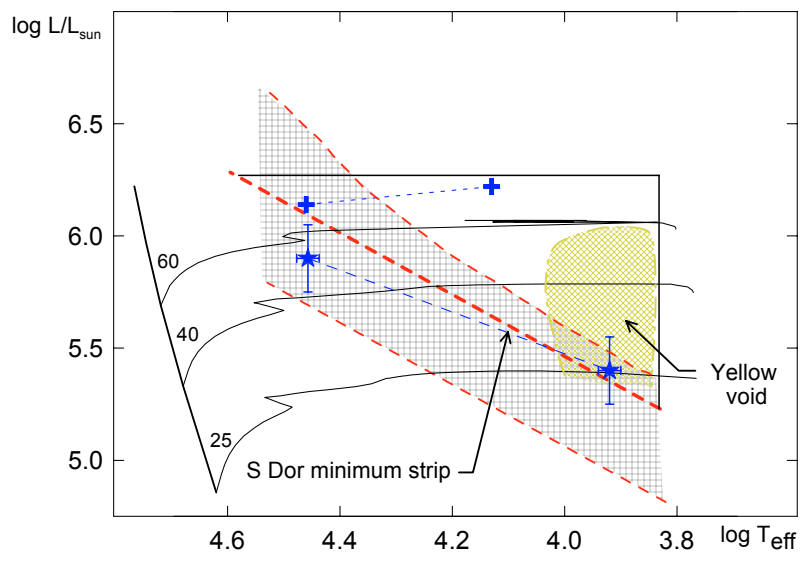

Fig. 5. The structure of the S Dor area on the HR-diagram (based in Fig. 21 of van Genderen 2001), with the S Dor-triangle, the S Dor minimum strip (fat dashed line) with the thinly dashed lines as its bandwidth, and the Yellow Void (de Jager \& Nieuwenhuijzen 1997; de Jager 1998). The extreme positions of Wra 751 ( $\star$ : left position 1989 , right position 2008) and AG Car (+) are indicated. The error bars give 1- $\sigma$ errors. Evolutionary tracks for 25,40 , and $60 M_{\odot}$ are shown.

40, and $60 M_{\odot}$ (Lejeune \& Schaerer 2001, case $e$, with mass loss, no rotation, $Z=0.001)$.

The uncertainties of both positions of Wra 751 are larger than the indicated nominal error bars because these positions rely on the rather uncertain $A_{V}=5.6$, and because there is no way to disentangle the interstellar, circumstellar, and dust extinction components of this extremely reddened star without a thorough spectroscopic analysis. Note the remarkable magnitude of the luminosity decrease during this leg of the LBV excursion in the HR diagram; although such a strong effect is also seen in the case of $\mathrm{S}$ Dor itself, where a range of $\Delta \log L / L_{\odot}=0.34$ corresponds to a variation in $\Delta \log T_{\text {eff }}=0.61$ (Table 1 of van Genderen 2001); see also Fig. 3 in Sterken (2003).

\section{Conclusions}

Multicolor photometry indicates that at this very moment the LBV Wra 751 is going through the bright (and red) stage of a long-term S Dor cycle, establishing the classification of Wra 751 as a "strong-active" S Dor variable (van Genderen 2001). The $S$ Dor behavior of this system closely resembles the characteristics of the Galactic LBV AG Car: the time scales and amplitudes of light and color variability are very similar.

The principal observational questions that remain unanswered, at this moment, concern the duration of the S Dor cycle and the exact atmospheric parameters of the star in this stage. This system evidently needs regular spectroscopic and photometric monitoring during the next full year, or at least until it returns to quiescence.

Acknowledgements. Based on observations made with the Danish $50 \mathrm{~cm}$ telescope (Strömgren Automatic Telescope, SAT) at the European Southern Observatory, La Silla, Chile. The telescope is operated by the Astronomical Observatory, the Niels Bohr Institute, Copenhagen University, Denmark. This research has made use of the ASAS, SIMBAD, and NASA ADS data bases.

This work was supported by the Research Foundation Flanders (FWO). The authors are indebted to Dr. G. Pojmánski for retrieving the ASAS data and to Dr. E. H. Olsen for SAT data support. We thank the referee, Dr. L. Drissen, for useful comments.

\section{References}

Appenzeller, I. 1972, PASJ, 24, 483

Ardeberg, A. 1980, A\&AS, 42, 1

Ardeberg, A., \& Maurice, E. 1977, A\&AS, 30, 261

Ardeberg, A., Brunet, J. P., Maurice, E., \& Prevot, L. 1972, A\&AS, 6, 249

Azzopardi, M., Vigneau, J., \& Macquet, M. 1975, A\&AS, 22, 285

Brunet, J. P., Prevot, L., Maurice, E., \& Muratorio, G. 1973, A\&AS, 9, 447

Caldwell, J. A. R., Cousins, A. W. J., Ahlers, C. C., van Wamelen, P., \& Maritz,

E. J. 1993, SAAO Circ., 15, 1

Clark, J. S., Larionov, V. M., \& Arkharov, A. 2005, A\&A, 435, 239

Crawford, D. L., Barnes, J. V., \& Golson, J. C. 1970, AJ, 75, 624

Crawford, D. L., Barnes, J. V., Hill, G., \& Perry, C. L. 1971a, AJ, 76, 1048

Crawford, D. L., Barnes, J. V., \& Golson, J. C. 1971b, AJ, 76, 1058

Crawford, D. L., Barnes, J. V., \& Golson, J. C. 1971c, Mon. Notes Astron. Soc. S. Afr., 30, 37

Dachs, J. 1970, A\&A, 9, 95

Dachs, J., Kaiser, D., Sherwood, W. A., \& Nikolov, A. 1982, A\&AS, 50, 261

Deutschman, W. A., Davis, R. J., \& Schild, R. E. 1976, ApJS, 30, 97

de Jager, C. 1998, A\&ARv, 8, 145

de Jager, C., \& Nieuwenhuijzen, H. 1997, MNRAS, 290, L50

Feast, M. W., Thackeray, A. D., \& Wesselink, A. J. 1960, MNRAS, 121, 337

Feinstein, A. 1968, Z. Astrophys., 68, 29

Feinstein, A. 1969, MNRAS, 143, 273

Feinstein, A., \& Ferrer, O. E. 1968, PASP, 80, 410

Feinstein, A., \& Marraco, H. G. 1979, AJ, 84, 1713

Fernie, J. D. 1983, ApJS, 52, 7

Flower, P. J. 1996, ApJ, 469, 355

Gray, R. O., \& Olsen, E. H. 1991, A\&AS, 87, 541

Grönbech, B., \& Olsen, E. H. 1976, A\&AS, 25, 213

Henize, K. G. 1976, ApJS, 30, 491

Hiltner, W. A. 1956, ApJS, 2, 389

Hogg, A. R. 1958, MtSOM, 2, 1

Hu, J. Y., de Winter, D., Thé, P. S., \& Perez, M. R. 1990, A\&A, 227, L17

Humphreys, R., \& Stanek, K. 2005, The Fate of the Most Massive Stars, ASPC, 332

Isserstedt, J. 1975, A\&AS, 19, 259

Johnson, H. L. 1964, Bol. Inst. Tonantzintla, 3, 305

Johnson, H. L. 1965, ApJ, 141, 923

Johnson, H. L., Iriarte, B., Mitchell, R. I., \& Wisniewskj, W. Z. 1966, Comm.

Lunar Plan. Lab, 4, 99

Jones, A., \& Sterken, C. 1997, JAD, 3,3

Kilkenny, D., \& Whittet, D. C. B. 1993, SAAOC, 15, 40

Kilkenny, D., Whittet, D. C. B., Davies, J. K., et al. 1985, SAAOC, 9, 55

Klare, G., \& Neckel, T. 1977, A\&AS, 27, 215

Knude, J. 1992, A\&AS, 92, 841

Kozok, J. R. 1985, A\&AS, 61, 387

Lejeune, T., \& Schaerer, D. 2001, A\&A, 366, 538

Lucke, P. B. 1973, Thesis

Manfroid, J., Oblak, E., \& Pernier, B. 1987, A\&AS, 69, 505

Mendoza, E. E. 1970, Bol. Tonantzintla, 5, 269

Nandy, K., Morgan, D. H., \& Houziaux, L. 1984, MNRAS, 211, 895

Nicolet, B. 1978, A\&AS, 34, 1

Olsen, E. H. 1994, A\&AS, 106, 257

Olsen, E. H. 2007, private communication

Osmer, P. S. 1973a, ApJ, 181, 327

Osmer, P. S. 1973b, ApJ, 186, 45

Pasquali, A., Comerón, F., \& Nota, A. 2006, A\&A, 448, 589

Pel, J. W. 1987, Internal Report, Leiden Observatory

Schild, R. E., Hiltner, W. A., \& Sanduleak, N. 1969, ApJ, 156, 609

Shobbrook, R. R. 1986, MNRAS, 219, 495

Shobbrook, R. R., \& Lynga, G. 1994, MNRAS, 269, 857

Sterken, C. 1977, A\&A, 57, 361

Sterken, C. 2003, Cycles and cyclicities in Luminous Blue Variables: the S Dor phenomenon, ASPC, 292, 437

Sterken, C., Freyhammer, L., Arentoft, T., \& van Genderen, A. M. 1999, A\&A, 346, L33

van Genderen, A. M. 2001, A\&A, 366, 508

van Genderen, A. M., Thé, P. S., de Winter, D., et al. 1992, A\&A, 258, 316

van Genderen, A. M., Sterken, C., \& de Groot, M. 1997, A\&A, 318, 81

Vigneau, J., \& Azzopardi, M. 1982, A\&AS, 50, 119

Walraven, Th., \& Walraven, J. H. 1964, Mon. Notes Astron. Soc. S. Afr., 23, 175

Westerlund, B. 1959, PASP, 71, 156

Wray, J. D. 1966, Ph.D. Thesis, North Western University 